\title{
DEPDC5 takes a second hit in familial focal epilepsy
}

\author{
Matthew P. Anderson \\ Boston, Massachusetts, USA. Program in Neuroscience, Harvard Medical School, Boston, Massachusetts, USA.
}

Departments of Neurology and Pathology, Beth Israel Deaconess Medical Center, Boston, Massachusetts, USA. Boston Children's Hospital Intellectual and Developmental Disabilities Research Center,
These detailed genetic and biochemical correlations in the human tissues then led them to design tests of causality in a mouse model, in which they reconstituted many features of the human disorder. Together, the results of Ribierre et al. provide compelling evidence to support the conclusion that focal epilepsy arising from DEPDC5 heterozygous mutations occurs through a second-hit loss of function of the second allele, as occurs in tuberous sclerosis.

The mouse model developed by Ribierre and colleagues allowed for further investigation into the cellular and molecular mechanisms of DEPDC5 mutationdriven focal epilepsy (7). The authors used an elegant approach of in vivo, in utero electroporation to deliver a CRISPR-Cas9 vector that allowed targeted homozygous deletions of Depdc5 alleles in cortical neuronal progenitor cells of the subventricular zone of fetal mouse brains. This targeted deletion led to deficits in neuronal migration, which were rescued by the treatment of pregnant dams with the mTORC1 inhibitor rapamycin. Moreover, neurons with homozygous Depdc5 inactivation had elevated levels of phosphorylated S6, which is indicative of mTORC1 activation, and increased soma size. These observations in the murine neurons recapitulated the findings in the human seizure resections (Figure 1). Importantly, mice with focal cortical mosaic homozygous deletions of Depdc5 suffered seizures, recorded by EEG, with tonic-clonic posturing in some cases and sometimes ending in seizure-related spontaneous death (a potential focal seizure-induced sudden death in epilepsy [SUDEP] model).

\section{Conclusions}

The evidence that pathogenic somatic mutagenesis is a cause of neurologic disorders is rapidly growing. Many genes involved in the mTOR and related signaling pathways have now been identified in focal and hemimegalencephaly cortical dysplasia cases (8-12). For many of the identified genes, it remains unclear exactly 


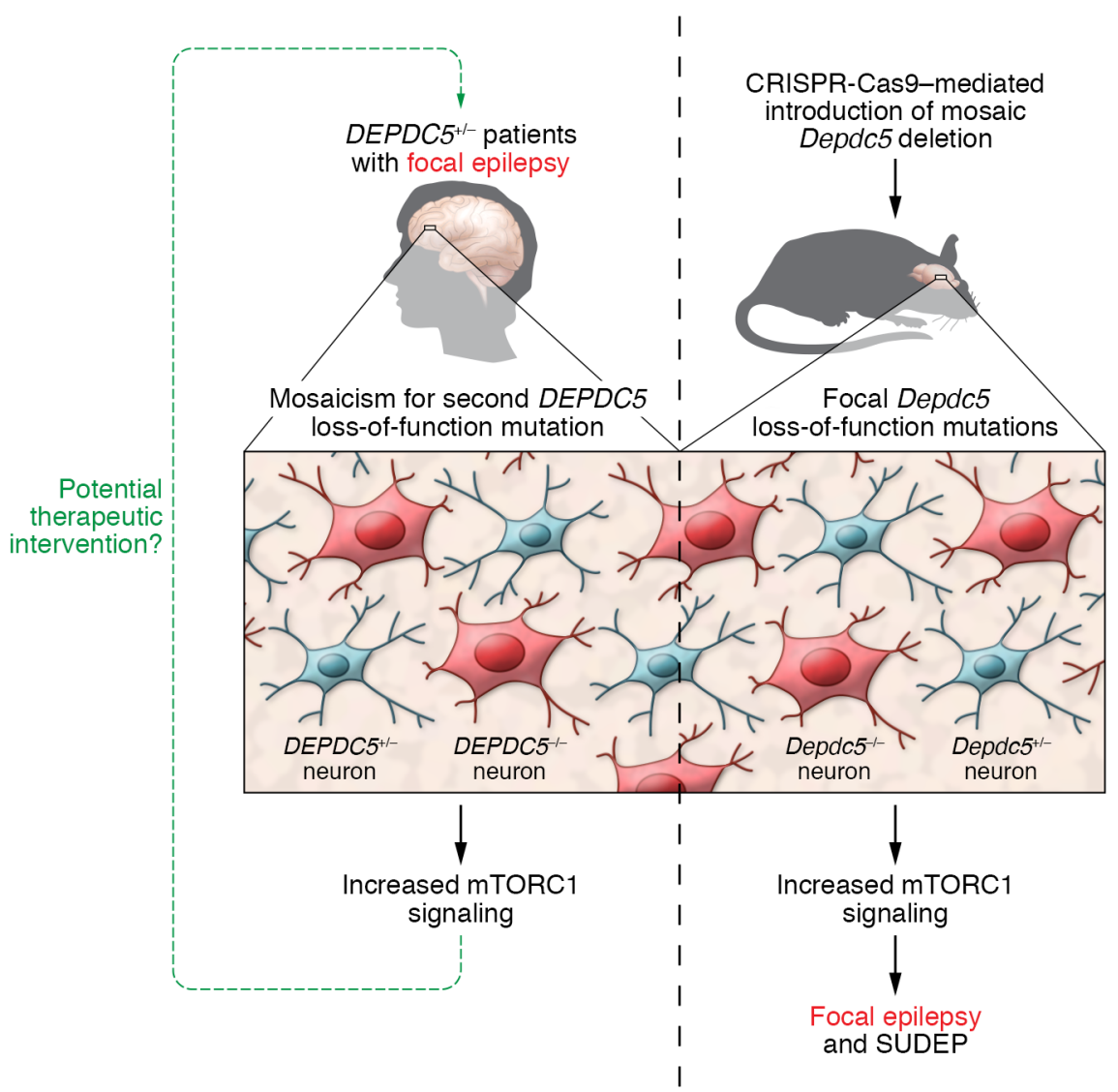

Figure 1. Second-hit DEPDC5 mutation underlies focal cortical dysplasia in familial epilepsy. In this issue, Ribierre et al. reveal a mosaic pattern of secondary somatic mutations in DEPDC5 in cortical neurons of $D E P D C 5^{-/+}$patients with focal epilepsy. The introduction of mosaic Depdc5 $5^{-/-}$mutations in a murine model recapitulates the patient phenotype, including magnified mTOR signaling, focal seizures, and SUDEP in some cases.

how these defects lead to epileptiform discharge of the affected neuronal circuits to produce the seizure foci; however, defects in cortical neuron migration, neuronal dendritic and axonal arbor overgrowth, and possibly alteration of the intrinsic excitability of the neurons carrying these mutations may alter the discharge of these dysplastic neuronal circuits. Interestingly, activation of the mTOR signaling pathway, via Pten deletion, in just a minor subset of neurons maturing in the adult dentate gyrus is sufficient to generate epilepsy (13).

Beyond mutations in growth and metabolism regulatory genes, loss of one allele of the Scn1a sodium channel in the GABAergic neurons is sufficient to promote severe seizures in mouse models of human Dravet syndrome. These seizures are presumably the result of an impaired ability of GABAergic neurons to sustain the high firing rates needed to inhibit circuit activity (14-16). A recent study also found evidence of somatic mutations of SCN1A and other genes in autism-epilepsy comorbidity disorders $(17,18)$. These preliminary results suggest the possibility that genes involved in neurophysiologic functions that would not produce a visible lesion might

Considering the now well-established evidence that somatic mutations in neuronal precursor cells can generate hyperexcitable circuits to produce epilepsy, it is readily conceivable that similar strategically placed mutations in neuronal precursors that give rise to circuits important to cognition and behavior could produce an array of episodic behavioral disorders such as panic, aggression, pain, or psychosis (19). Moreover, strategically placed seizure foci might also generate lasting changes in behavior, such as the impaired sociability of autism spectrum disorder, by altering the expression of genes critical to circuit functions (20). also underlie some seizure foci.

\section{Acknowledgments}

MPA receives funding from the National Institute of Neurological Disorders and Stroke (NINDS) (1R01NS08916); the National Institute of Mental Health (1R01MH114858; 1R01MH112714); the Nancy Lurie Marks Family Foundation; the Landreth Foundation; Autism Speaks/ National Alliance for Autism Research; and the Simons Foundation. Support is also provided to the Neurobiology Department of Harvard Medical School (in part through the NINDS P30 Core Center grant NS07203) and to the Boston Children's Hospital Intellectual and Developmental Disabilities Research Center (IDDRC) (1U54HD090255 and P3OHD18655).

Address correspondence to: Matthew P. Anderson, Center for Life Science, 330 Brookline Ave, E/CLS-645, Boston, Massachusetts 02215, USA. Phone: 617. 735.3202; Email: Matthew_Anderson@ bidmc.harvard.edu.

1. Scheffer IE, et al. Familial partial epilepsy with variable foci: a new partial epilepsy syndrome with suggestion of linkage to chromosome 2. Ann Neurol. 1998;44(6):890-899.

2. Dibbens LM, et al. Mutations in DEPDC5 cause familial focal epilepsy with variable foci. Nat Genet. 2013;45(5):546-551.

3. Ishida S, et al. Mutations of DEPDC5 cause autosomal dominant focal epilepsies. Nat Genet. 2013;45(5):552-555.

4. Scheffer IE, et al. Mutations in mammalian target of rapamycin regulator DEPDC5 cause foca epilepsy with brain malformations. Ann Neurol. 2014;75(5):782-787.

5. Baulac S, et al. Familial focal epilepsy with focal cortical dysplasia due to DEPDC5 mutations. Ann Neurol. 2015;77(4):675-683.

6. Bar-Peled L, et al. A Tumor suppressor complex with GAP activity for the Rag GTPases that signal amino acid sufficiency to mTORC1. Science. 2013;340(6136):1100-1106.

7. Ribierre T, et al. Second-hit mosaic mutation in mTORC1 repressor DEPDC5 causes focal cortical dysplasia-associated epilepsy. J Clin Invest. 2018;128(6):2452-2458.

8. Poduri A, et al. Somatic activation of AKT3 causes hemispheric developmental brain malformations. Neuron. 2012;74(1):41-48.

9. Lee JH, et al. De novo somatic mutations in components of the PI3K-AKT3-mTOR pathway cause hemimegalencephaly. Nat Genet. 2012;44(8):941-945.

10. Baek ST, et al. An AKT3-FOXG1-reelin network underlies defective migration in human focal malformations of cortical development. Nat Med. 2015;21(12):1445-1454.

11. Ricos MG, et al. Mutations in the mammalian target of rapamycin pathway regulators NPRL2 
and NPRL3 cause focal epilepsy. Ann Neurol. 2016;79(1):120-131.

12. D'Gama AM, et al. Somatic mutations activating the mTOR pathway in dorsal telencephalic progenitors cause a continuum of cortical dysplasias. Cell Rep. 2017;21(13):3754-3766.

13. Pun RY, et al. Excessive activation of mTOR in postnatally generated granule cells is sufficient to cause epilepsy. Neuron. 2012;75(6):1022-1034.

14. Yu FH, et al. Reduced sodium current in GABAergic interneurons in a mouse model of severe myoclonic epilepsy in infancy. Nat Neurosci.
2006;9(9):1142-1149.

15. Han S, et al. Autistic-like behaviour in Scn1a ${ }^{+/}$mice and rescue by enhanced GABA-mediated neurotransmission. Nature. 2012;489(7416):385-390.

16. Cheah CS, et al. Specific deletion of NaV1.1 sodium channels in inhibitory interneurons causes seizures and premature death in a mouse model of Dravet syndrome. Proc Natl Acad Sci U S A. 2012;109(36):14646-14651.

17. D'Gama AM, et al. Targeted DNA sequencing from autism spectrum disorder brains impli- cates multiple genetic mechanisms. Neuron 2015;88(5):910-917.

18. Krupp DR, et al. Exonic mosaic mutations contribute risk for autism spectrum disorder. $\mathrm{Am} J$ Hum Genet. 2017;101(3):369-390.

19. McConnell MJ, et al. Intersection of diverse neuronal genomes and neuropsychiatric disease: The Brain Somatic Mosaicism Network. Science. 2017;356(6336):eaal1641.

20. Krishnan V, et al. Autism gene Ube3a and seizures impair sociability by repressing VTA Cbln1. Nature. 2017;543(7646):507-512. 